\title{
BMJ Open Quality Improving the quality of assessment and management of nasal trauma in a major trauma centre (MTC): Queen Elizabeth Hospital, Birmingham
}

\author{
Apoorva Khajuria (D) , ${ }^{1}$ Max Sallis Osborne (D) , ${ }^{1}$ Lisha McClleland, ${ }^{1}$ Sandip Ghosh ${ }^{2}$
}

To cite: Khajuria A, Osborne MS, McClleland L, et al. Improving the quality of assessment and management of nasal trauma in a major trauma centre (MTC): Queen Elizabeth Hospital, Birmingham. BMJ Open Quality 2019;8:e000632. doi:10.1136/ bmjoq-2019-000632

- Additional material is published online only. To view please visit the journal online (http://dx.doi.org/10.1136/ bmjoq-2019-000632).

Received 10 January 2019 Revised 5 November 2019 Accepted 9 November 2019

\section{Check for updates}

(c) Author(s) (or their employer(s)) 2019. Re-use permitted under CC BY-NC. No commercial re-use. See rights and permissions. Published by BMJ.

${ }^{1}$ Ear, Nose \& Throat (ENT) Department, Queen Elizabeth Hospital Birmingham,

Birmingham, UK

${ }^{2}$ Diabetes and Endocrinology Department, Queen Elizabeth Hospital Birmigham, Birmingham, UK

Correspondence to Dr Apoorva Khajuria; ap.khajuria@gmail.com

\section{ABSTRACT}

Background Nasal fractures present in $39 \%$ of patients with facial trauma. These patients are assessed in the emergency department followed by outpatient review in the senior house officer-led emergency ear, nose and throat (ENT) clinic. Inadequate treatment of nasal trauma can result in debilitating functional and aesthetic problems. Inexperienced junior doctors may be apprehensive in assessing nasal trauma resulting in time pressured clinics and suboptimal management.

Measures A retrospective review of clinical noting over 3 months was carried out to gauge the extent of the problem. Three baseline measurements for satisfactory quality of assessments included: (1) 3/5 key symptoms elicited by the clinician (epistaxis, rhinorrhoea, nasal airway obstruction, dental malocclusion and diplopia). (2) Presence/absence of 'septal haematoma' (SH) and 'deviated nasal septum' (DNS) documented. (3) Patient follow-up within 2 weeks after the initial injury. Three Plan-Do-Study-Act (PDSA) cycles were conducted with implementation of interventions (proforma, clinic poster, patient information leaflet and training) as visualised in our 'driver diagram'.

Results The quality of nasal trauma assessments improved following each intervention. There was an increase from $86 \%$ to $100 \%$ patients being seen within 2 weeks of the injury. There was an improvement in quality of assessments following the teaching as two-thirds (PDSA cycle 2) followed by $100 \%$ (PDSA cycle 3) of clinical documentation included 'rhinorrhoea' and 83\% (PDSA cycle 2) to $100 \%$ (PDSA cycle 3) included 'nasal airway obstruction'. Similarly, two thirds (PDSA cycle 2) followed by $100 \%$ (PDSA cycle 3 ) examined and documented the presence/absence of SH and DNS. A 100\% improvement in trainee confidence was reported. We are now conducting more comprehensive assessments of nasal trauma patients.

Conclusion The need to provide relevant training and support to ENT junior doctors is crucial in their development, as well as to ensure delivery of high-quality patient-centred care.

\section{PROBLEM DESCRIPTION}

This improvement activity took place in a busy Ear, Nose and Throat (ENT) department within a 1213-bed major trauma centre in the UK. Presently, there is limited evidence and no specific guidelines to provide a standard of care for nasal fracture patients. Therefore, junior doctors with little prior ENT experience may find themselves perplexed when assessing such injuries.

Violent crime and antisocial behaviour in Birmingham is on the rise; we found assault and violence the most common causes of nasal injuries in our patient cohort. ${ }^{1}$ The ENT senior house officer (SHO) on-call would book nasal trauma patients into the SHOled emergency clinic for further assessment or management; these clinics occurred daily and each appointment lasted 30 minutes. SHOs found nasal trauma patients difficult to assess as they were unsure of what constitutes 'comprehensive' assessment and if management such as a manipulation under anaesthesia (MUA) was required which would then require senior input thus adding time-pressure to an already busy clinic. Care was not standardised, thus, the project team designed assessment tools to enable junior doctors to carry out more robust assessments of nasal injuries and standardise care.

\section{BACKGROUND}

Nasal fractures are the most common type of facial fractures and frequently present to the emergency department (ED).$^{2-5}$ They can be part of more serious facial injuries or an isolated injury following physical altercations. Regardless of the nature of the injury, correct assessment and management must be carried out for all patients to avoid complications and provide good aesthetic outcomes. ${ }^{67}$ Key issues surrounding inexperienced junior doctors assessing this patient cohort are: unnecessary investigations, inadequate documentation and inappropriate acute management or follow-up arrangements. Another example of insufficient assessment includes overlooking the need to differentiate an acute deformity 
from a pre-existing chronic deformity as this will make a MUA difficult thereby altering management. ${ }^{8}$

There are presently no robust, user-friendly tools that exist to allow standardised and efficient assessment and management of nasal trauma patients thus presenting a challenge for junior doctors in deciding how to approach the patient. In our hospital, this resulted in a registrar being contacted to assist in clinic. If registrars were readily available, then it became a possible training opportunity as per Kolb's experiential learning cycle, although the value of experiential learning is limited without appropriate reflection and conceptualisation. ${ }^{9}$ It is important to note that due to time pressures and the need to prioritise patient safety, Halsted's 'see one, do one, teach one' model may no longer be an appropriate method for practical skills training. ${ }^{910}$ If registrars were not immediately available to assist in clinic then this caused clinic delays and patient frustration.

There is limited evidence to suggest an accurate time for nasal trauma patient follow-up, however, the general consensus among experts is reassessment within 7-10 days (ideally within a 2-week window) from the day of injury. ${ }^{11-13}$ - The reason for the delay in re-reviewing patients is to allow oedema to subside, at which point nasal deformities are easier to assess and closed reduction can be considered. ${ }^{14}$ Finally, clinician training and patient education may be essential tools to optimise clinic appointments. ${ }^{15}$ For example, predesigned proformas aid in diminishing errors due to human factors in time pressured situations. ${ }^{16}$

\section{DESIGN}

The need for a sustainable assessment tool was apparent to enable junior doctors to better assess patients and so a proforma was developed by a team consisting of a SHO, Registrar and an ENT consultant. We aimed to improve the quality of care for nasal trauma patients in the ENT SHO-led emergency clinic to ensure timely management, accurate assessment and documentation of key symptoms and examination findings during all nasal trauma consultations. The proforma could be used flexibly in the form of a hard copy which is then scanned into 'clinical portal' (our local patients' electronic records system) or as a framework to document consultations in the traditional clinical noting system; this made it a simple and sustainable tool.

\section{Context}

The patient group was selected by identifying patients with suspected nasal fracture who had been booked into the SHO-led emergency clinic between June and August 2017. Patients were booked into this clinic following:

i. Direct review by the ENT on-call SHO in ED or

ii. Direct review by an ED doctor who contacts the ENT SHO over the phone to request an emergency ENT clinic appointment.

Initial data collection involved retrospective review of emergency ENT clinic noting of nasal trauma patients to gauge the extent of the problem. A data spreadsheet was created to capture objective related baseline measurements. Over the 3 months period, 58 nasal trauma patients were identified. Out of 58 patients, one was seen in Maxillo-facial (MaxFacs) clinic due to extent of injuries. 14 patients did not attend their appointments and so a total of 43 clinic notes were reviewed to establish baseline measurements. The implementation of the tools (see below) developed from this project, specifically the proforma, was monitored. During the project, new junior doctors joined the team, they were educated and signposted to the proforma and other tools by senior members of the team. Additionally, the project was presenting again, 6 months following the initial work.

\section{Interventions}

Proforma

A driver diagram (figure 1) was used to develop additional ideas for our improvement strategy, including the assessment proforma. The proforma documents the date, specific time the clinic appointment started and patient demographics. The time aspect was important as we postulated that nasal trauma patients, due to the nature of their injury, would be more time consuming especially if an inexperienced junior doctor rightfully contacted the

\section{Driver diagram}

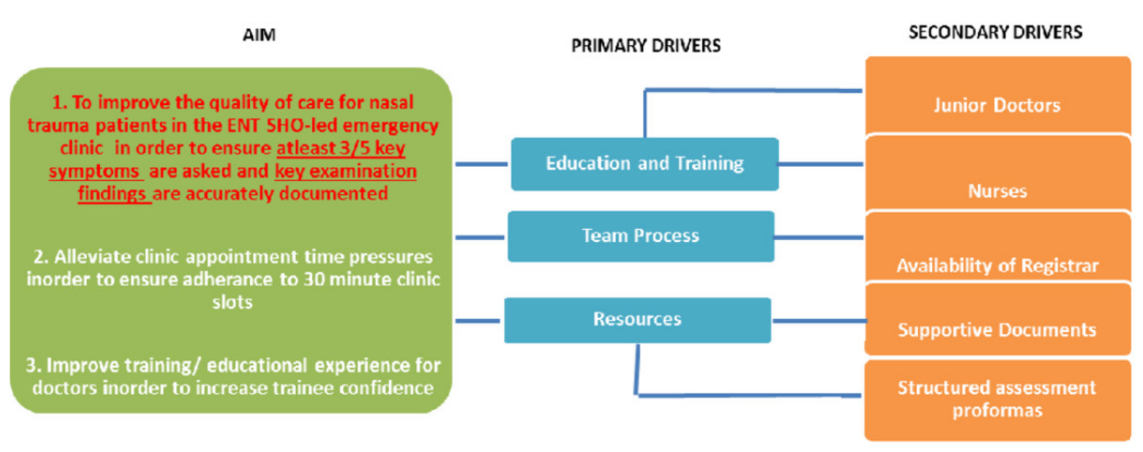

CHANGE IDEAS

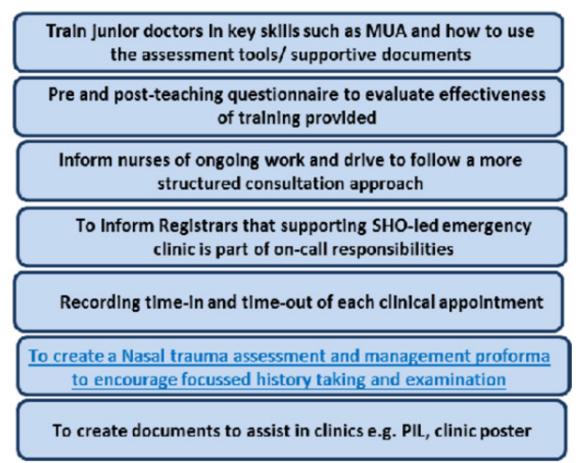

Figure 1 Driver diagram showing the development of intervention ideas. ENT, ear, nose and throat; MUA, manipulation under anaesthesia; PIL, patient information leaflet; SHO, senior house officer. 
Registrar for assistance. The proforma then asks about nasal trauma in the past as this can significantly increase the difficulty of the reduction, if needed, due to previously healed fracture. The nose is a crucial aesthetic component to the face-based on this and discussions with patients we incorporated 'aesthetic perception' in the assessment proforma. To supplement this, the proforma suggests reviewing an old photograph to assess the nasal profile of the patient, if available and time permitting (see online supplementary file).

Key associated symptoms are tabulated for selection by circling the applicable symptom and further investigations, if relevant, are recommended. For example, if a patient complains of clear, watery nasal discharge, the proforma suggests considering a B-transferrin sample to test for cerebrospinal fluid (CSF). The second page of the proforma focuses primarily on the examination features based on external examination and internal rhinoscopy. Finally, the 'plan' section of the proforma documents consent and if any procedures were carried out. A brief patient education table prompts clinicians to provide key post-injury management information. Finally, the clinician signs and dates the proforma with documentation of the time the patient left the clinic room.

\section{Other Interventions}

Additional interventions were developed during the project to further enhance the patient experience and improve team morale and confidence. A registrar-led nasal trauma teaching session was arranged for all ENT junior doctors. The session covered nasal anatomy, managing referrals, key aspects of assessment, carrying out a MUA and legality issues surrounding nasal injuries. Junior doctors were also educated on use of the proforma. Presession and post-session questionnaire data was collated. The expert group designed and created a clinic poster titled: 'MUA of Nasal Bones under Local Anaesthetic'. This was formatted in a clear flowchart arrangement and displayed in clinic as a quick reference guide. However, SHOs were still encouraged to call the on-call registrar if they required extra support or did not feel comfortable carrying out a MUA. A specialist group of two consultant Rhinologists were asked to independently review the patient information leaflet (PIL) which was developed for clinic. Prior to our project, no PIL existed for patient education on nasal injuries. Once the content was proofchecked, the leaflet was approved and processed by the Patient Information Group (see online supplementary files $2 \mathrm{a}$ and $2 \mathrm{~b}$ ).

\section{Measures}

Baseline measurement 1: to assess history taking and documentation

We classified consultations where at least 3/5 key symptoms (epistaxis, rhinorrhoea, nasal airway obstruction, dental malocclusion and diplopia) were asked by the clinician to be deemed satisfactory. These symptoms were selected based on the research team's clinical knowledge, experience and was in keeping with available evidencethe presence of these symptoms may indicate the need for further investigation and management of the nasal trauma. ${ }^{1718}$

Rhinorrhoea and airway obstruction had to constitute two of the symptoms elicited otherwise the history was deemed unsatisfactory; this is because airway obstruction is an indication for formal rhinoplasty under the National Health Service (NHS) and therefore important to establish during consultation and CSF rhinorrhoea is a serious complication of craniofacial fractures. ${ }^{19} \mathrm{Up}$ to $80 \%$ of patients with CSF rhinorrhoea have an associated facial fracture and are at risk of infections such as Meningitis. ${ }^{20}$

Thus, it is crucial to ask patients if they have noticed any 'watery nasal discharge' and if a sample was collected. If so, the sample can be tested for beta-transferrin which would indicate the presence of CSF. We found that only six clinic notes had documented asking at least $3 / 5$ key question in the history and only two of these consultation notes included both rhinorrhoea and nasal airway obstruction.

\section{Baseline measurement 2: to assess examination findings and documentation}

The clinic notes were evaluated to assess the quality of nasal examinations. The team identified septal haematoma ( $\mathrm{SH})$ and deviated nasal septum (DNS) as crucial examination findings, without which the consultation would be deemed unsatisfactory. This is because a $\mathrm{SH}$ requires rapid evacuation to prevent an abscess/necrosis which, subsequently, causes nasal collapse and deformity. The presence of DNS has been shown to be a predictor of failure of closed reduction and need for revision septorhinoloplasty therefore it is important to document and counsel the patient appropriately. ${ }^{21} 12 / 43(28 \%)$ clinical documentation included both SH and DNS. Some noting was ambiguous and used words such as cartilage which may refer to alar cartilage or septal cartilage therefore these were considered unsatisfactory.

\section{Baseline measurement 3 : to assess timely management}

Patients' presenting time to the ENT SHO-led clinic after the injury ranged between 3 and 24 days (mean 10.1 days) which is beyond the optimal 2-week window of following injury. 37 patients $(86 \%)$ were seen within 2 weeks of injury. We widely disseminated the above findings via email and through presentation at the monthly ENT departmental meeting. Following discussions, the nasal trauma proforma was developed for use in clinic. We continued to measure the quality of assessments against the proforma and developed additional tools based on the data collected. We wanted to objectively assess clinic appointment time pressures by noting the time-in and time-out of each clinical appointment. Unfortunately, we found this was poorly documented and results were unrealistic. 


\section{STRATEGY}

\section{Plan-Do-Study-Act Cycle 1}

We aimed to improve the quality of care for nasal trauma patients in the ENT SHO-led emergency clinic to ensure timely management, accurate assessment and documentation of key symptoms and examination findings during all nasal trauma consultations through the proforma. The proforma was presented, along with current evidence and expert consensus around nasal injury management, in a monthly departmental meeting and was made available as open access to all ENT SHOs. As predicted, the proforma was well received in the department and junior doctors were keen to use it in the clinical setting.

\section{Plan-Do-Study-Act Cycle 2}

The aim of the second cycle was to further improve outcomes from proforma use through a registrar led teaching programme which was delivered to all SHOs. Pre-teaching and post-teaching questionnaire data showed an increase in trainee confidence in history taking and examination from $0 \%$ (very confident), to $100 \%$ (fairly-very confident). Junior doctors provided exceptional 'free text' feedback supporting the use of the proforma. Suggestions from the feedback highlighted that SHOs may benefit from additional tools such as a PIL and clinic poster on MUA. A fifth of SHOs reported that the outcomes from this project were contributing to off-load clinic time pressures; perhaps due to increased confidence in managing nasal trauma patients through training and guidance tools.

\section{Plan-Do-Study-Act Cycle 3}

We created a PIL and a 'Carrying out a MUA of Nasal Bones under Local Anaesthetic' poster. These tools were displayed in clinic rooms and circulated to all SHOs via email. The poster was a reference tool to assist clinicians already competent in carrying out MUA, otherwise junior doctors were encouraged to continue to seek advice from the on-call Registrar if needed. A reminder system was put in place in the form of a poster on the ward and doctors' office which showed how to navigate the computer system to locate the assessment tools.

The only issue that remained was the last section of the proforma ('patient education'). On discussion with SHOs it was felt that aspects of 'patient education' were inapplicable to some patients. For example, advice surrounding avoiding contact sport is inappropriate for an elderly patient with a nasal injury after a fall. This was taken into consideration and it was decided that patient education prompts would remain in the proforma but should be discussed on a patient-specific basis.

\section{RESULTS}

Plan-Do-Study-Act (PDSA) Cycle 1: with successful uptake of the proforma, we noted an increase from $86 \%$ to $100 \%$ patients being seen within 2 weeks of the injury. No improvement was noted in eliciting one of the key features (rhinorrhoea) in the history. However, for the other key feature (airway obstruction), 100\% of consultations documented 'airway obstruction' in the history. A quarter of clinic appointments recorded both DNS and SH in clinical noting, however, this was an improvement from baseline. No one documented the 'time in' or 'time out' of appointments however, all junior doctors did record the management outcome from which the clinic time pressures may be determined. PDSA Cycle 2: following the registrar led teaching programme, the number of patients seen within 2 weeks of their injury decreased from $100 \%$ in the previous cycle to $83 \%$. Detailed analysis showed that this was due to patient factors such as patients being unable to attend earlier appointments (within 2-week window period) due to planned commitments. Additionally, there was an improvement in quality of assessments following the teaching as $67 \%$ of clinical documentation now included 'rhinorrhoea' and 83\% included 'nasal airway obstruction'. Similarly, two-thirds of consultations included the presence/absence of $\mathrm{SH}$ and DNS. PDSA Cycle 3: At this stage, the quality of documentation was of a high standard. All consultations now included documentation of 'airway obstruction' and 'rhinorrhoea'. Documentation of all key symptoms listed in the proforma occurred in $100 \%$ of consultations; notably $75 \%$ of consultation included all five of the symptoms listed in the proforma.

\section{DISCUSSION}

\section{Summary}

Through this project we learnt to appreciate the value in continued evaluation of recommendations which ensured that recommendations were effectively utilised and provided maximum benefit. The quality of nasal trauma assessments improved significantly following each intervention. Outcomes showed that the proforma was effective in ensuring timely assessment of patients $(100 \%)$ in comparison to baseline data $(86 \%)$. Once clinicians had been trained in using the proforma, they formally reported being more confident in managing such patients in clinic. Clinical documentation became more comprehensive and included key features in the history and examination of these patients as set out in our aims (figure 2). The need to book patients into emergency clinic within a 2-week window was highlighted to junior doctor through subsequent PDSA cycles and this was reflected in practice.

\section{Interpretation}

There is a gap in the availability of guidelines/protocols for assessment and management of nasal trauma and the need for such guidance has already been highlighted in literature. We found that front line staff (junior doctors) promoted the implementation of our proforma and additional tools as guidelines for clinical encounters, specifically for use in the clinic setting. The support for these assessment tools has also been reflected in the improvements in outcome measures during this project. Although 


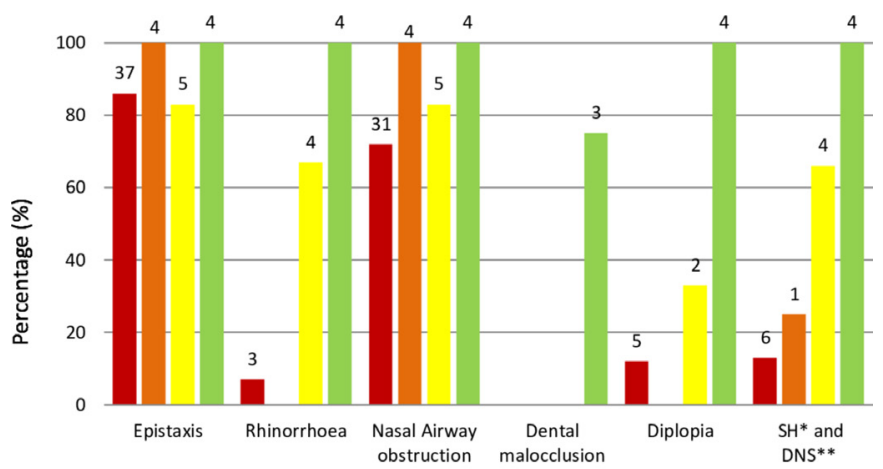

* Septal Haematoma

- Baseline $=$ PDSA 1 PDSA 2 -PDSA

Figure 2 Percentage of clinic appointments in which key clinical features identified from the history taking and examination were documented per PDSA cycle. PDSA, PlanDo-Study-Act.

not part of the formal measures, additional parameters demonstrated a $15 \%$ increase in discussions regarding the patient's aesthetic perception of their nose and an increase in the proportion of junior doctors enquiring about epistaxis (increase from $86 \%$ to 100\%). The observed outcomes were as anticipated with the quality of nasal trauma assessments improving significantly.

\section{Limitations}

Our primary outcome measure (quality of care) was challenging to interpret as it was multicomponent. A major limitation was that the duration of each clinic appointment was not accurately recorded. Therefore, the data was not suitable for interpretation. The reason for this is because the clinic computer system allows the clinician to select new patients without completing the 'finish form' of their current patient; therefore, often these forms are missed or completed at a later stage. This was discussed during our second PDSA cycle and it was thought that timing a clinic appointment is not always prioritised and it is sufficient to note improvement in clinic time pressure based on experiences of front-line staff. Thus, by improving efficiency by means such as education and provision of assessment tools, we may also overcome the issue of time pressured clinics. Finally, our outcome measures showed some variation that may be as a result of subjective interpretation of the proforma however we endeavoured to tackle this through the teaching programme.

Implementation challenges included: ensuring awareness and motivation among doctors to comply with recommendations and Patient Information Group authorisation to enable poster and PIL use. From this, we learnt that implementation of change is a slow process involving many steps and multiple teams. Discussion with key individuals (junior doctors) is useful in identifying and overcoming implementation barriers. A major barrier was the accessibility of the proforma which we overcame through displaying posters in the doctors' ward office and in clinic to guide doctors to the correct folder on the computers.
If this project was repeated, we would highlight the importance of time keeping to junior doctors from the start as incorrect documentation invalidated time keeping data. It is important to note that team culture and motivation are also key contributors to sustainability. The sample sizes for subsequent PDSA cycles would be greater by carrying out data collection over a longer period of time. Finally, we would appoint a 'quality improvement champion' for this project. This would be a registrar and the role would involve educating future SHOs to ensure key messages are retained and so that they may benefit from the recommendations from this work.

\section{CONCLUSIONS}

Nasal fractures are commonly assessed by ENT junior doctors; who acknowledged not feeling confident in assessing these patients, thereby potentially compromising patient safety as well as their clinical development. This project made a significant impact locally by achieving a $100 \%$ improvement in trainee confidence and generating more comprehensive patient assessments. We believe that through supportive management in the form of dedicated consultants and registrars and maintenance of team awareness we can ensure sustainability of our quality improvement work. The junior led ENT clinics provide an ample training opportunity and enable development of important skills such as decisionmaking and communication. However, training cannot be undertaken at the cost of patient safety therefore our recommendations optimise junior doctor training as well as equipping doctors with the necessary tools to ensure high-quality and safe management of patient.

Further work is required to evaluate long term outcomes (formal rhinoplasty) in this patient cohort and whether these patients underwent an operative MUA. Also, more formal/objective assessment of patient satisfaction and feedback would be useful to allow further improvements in our service. Despite moving onto different departments, we ensured this project was continued over the required length of time and we continue to educate and maintain the recommendations as described in this paper. We hope that through the platform of academic forums we can share our new method of practice so our peers may benefit from these innovative assessment tools.

Acknowledgements Everyone involved in the quality improvement work including patients, junior doctors, senior trainees, consultants and the wider multidisciplinary team for their ongoing support.

Contributors AK conceived the idea, conducted baseline measurements/ data collection; data analysis wrote and submitted the manuscript. MSO and LM supervised the findings of the work and critically reviewed the manuscript. SG developed the theoretical framework with AK and critically reviewed the manuscript.

Funding The authors have not declared a specific grant for this research from any funding agency in the public, commercial or not-for-profit sectors.

Competing interests None declared.

Patient consent for publication Not required.

Provenance and peer review Not commissioned; externally peer reviewed. 
Open access This is an open access article distributed in accordance with the Creative Commons Attribution Non Commercial (CC BY-NC 4.0) license, which permits others to distribute, remix, adapt, build upon this work non-commercially, and license their derivative works on different terms, provided the original work is properly cited, appropriate credit is given, any changes made indicated, and the use is non-commercial. See: http://creativecommons.org/licenses/by-nc/4.0/.

\section{ORCID iDs}

Apoorva Khajuria http://orcid.org/0000-0003-1679-1364

Max Sallis 0sborne http://orcid.org/0000-0001-6081-9307

\section{REFERENCES}

1 UK Crime Stats. West Midlands police. Available: http://www. ukcrimestats.com/Police_Force/West_Midlands_Police [Accessed 22nd Dec 2018].

2 Erdmann D, Follmar KE, DeBruijn M, et al. A retrospective analysis of facial fracture etiologies. Ann Plast Surg 2008;60:398-403.

3 Hwang K, You SH. Analysis of facial bone fractures: an 11-year study of 2,094 patients. Indian J Plast Surg 2010;43:42-8.

4 Peeters N, Lemkens P, Leach R, et al. Facial trauma. B-ENT 2016;26:1-18.

5 Louis M, Agrawal N, Kaufman M, et al. Midface fractures I. Semin Plast Surg 2017;31:85-93

6 Kelley BP, Downey CR, Stal S. Evaluation and reduction of nasal trauma. Semin Plast Surg 2010;24:339-47.

7 Renner GJ. Management of nasal fractures. Otolaryngol Clin North Am 1991;24:195-213.
8 Ferguson G, Bell PR, Hall SJ. What becomes of the broken nose? Ulster Med J 2014;83:49-50.

9 Kotsis SV, Chung KC. Application of the "see one, do one, teach one" concept in surgical training. Plast Reconstr Surg 2013;131:1194-201.

10 Hurreiz $\mathrm{H}$. The evolution of surgical training in the UK. Adv Med Educ Pract 2019;10:163-8.

11 ENT UK. Nasal injuries. Available: https://www.entuk.org/nasalinjuries-0. [Accessed 14th Aug 2019].

12 Koh JH, Bhatti O, Mahmood A, et al. Traumatic nasal injuries in general practice. Aust Fam Physician 2016;45:650-3.

13 Yoon HY, Han DG. Delayed reduction of nasal bone fractures. Arch Craniofac Surg 2016;17:51-5.

14 Thiagarajan B, Ulaganathan V. Fracture nasal bone and its management. Otolaryngology 2013;3:1.5

15 Coulter A, Ellins J. Effectiveness of strategies for informing, educating, and involving patients. BMJ 2007;335:24-7.

16 Patient safety UK. North East strategic health authority patient action team human factors for healthcare. Available: http://patientsafety. health.org.uk/sites/default/files/human_factors_in_healthcare_trainer manual_en_march_2013.pdf [Accessed 22nd Dec 2018].

17 Tasman A-J. Rhinoplasty - indications and techniques. GMS Curr Top Otorhinolaryngol Head Neck Surg 2007;6:Doc09.

18 Rudy SF, Most SP. Rhinoplasty - indications and techniques. JAMA 2017;318:1406.

19 Phillips BJ, Turco LM. Le Fort fractures: a collective review. Bull Emerg Trauma 2017;5:221-30.

20 Oh J-W, Kim S-H, Whang K. Traumatic cerebrospinal fluid leak: diagnosis and management. Korean J Neurotrauma 2017;13:63-7.

21 Ondik MP, Lipinski L, Dezfoli S, et al. The treatment of nasal fractures: a changing paradigm. Arch Facial Plast Surg 2009;11:296-302. 